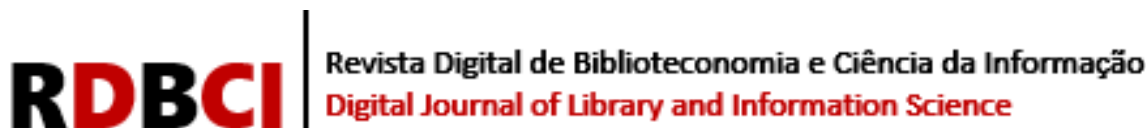

\section{$\mathbf{I S N}_{1678-765 \mathrm{X}}$ do ${ }_{10.20396 / \mathrm{rdbci} . \mathrm{v} 18 \mathrm{io} .8666250}$ (c) (i) $\mathrm{BY}$ \\ Dinâmicas da produção científica brasileira em revistas da área de Comunicação na Web of Science}

ARTIGO DE PESQUISA

\author{
Elaine Rosangela de Oliveira Lucas ${ }^{1}$ (iD http://orcid.org/0000-0002-2796-3566 \\ Guadalupe Aguado-Guadalupe 2 (iD) https://orcid.org/0000-0003-0367-0299 \\ Eva Herrero-Curiel ${ }^{3}$ (D) https://orcid.org/0000-0003-0801-2519 \\ ${ }^{1}$ Universidade do Estado de Santa Catarina, Florianópolis, SC, Brasil / e-mail: Ianilucas@gmail.com \\ 2 Universidad Carlos III de Madrid, Marília, SP, Brasil / e-mail: maguado@hum.uczm.es \\ ${ }^{3}$ Universidad Carlos III de Madrid, Madri, Espanha / e-mail: eherrero@hum.uczm.es
}

\section{RESUMO}

Introdução: Tendo em consideração que a atividade de investigação em Comunicação cresceu fortemente nos últimos anos, como indicado por estudos anteriores, o presente trabalho visa estabelecer uma pesquisa em termos de produção científica nessa área. Objetivos: Esse artigo analisa a produção científica na área da Comunicação, entre 2007 e 2017, em revistas indexadas no primeiro quartil (Q1) e no segundo quartil (Q2) da base de dados Web of Science, pertencentes a autores com afiliação em instituições brasileiras. Metodologia: Foram identificadas as revistas de comunicação no InCities (JCR) pertencentes aos dois primeiros quartis, em seguida foi realizada uma busca por título de periódico na WoS e posteriormente o tratamento e normalização dos dados obtidos. Resultados: Observa-se um crescimento na produção científica em Comunicação, na projeção internacional, alimentando uma tradição em coautoria com redes internacionais. No período de 2014 a 2017, tenderam a publicar principalmente em inglês, frente às colaborações usuais que vinham mantendo com países de língua espanhola até 2014. Conclusão: Conclui-se, entre outras coisas, que o sistema de avaliação da Capes, quadrienal, pode ter influenciado a produção científica em Comunicação no intervalo estudado.

PALAVRAS-CHAVE

Comunicação. Produção científica. Revistas de comunicação. Web of Science. Brasil.

\section{Dynamics of Brazilian scientific production in Communication journals in the Web of Science}

\begin{abstract}
Introduction: Taking into consideration that research activity in Communication has grown strongly in recent years, as indicated by previous studies, the present work aims to establish a survey in terms of scientific production in this area. Objectives: This paper analyzes the scientific production in the area of Communication, between 2007 and

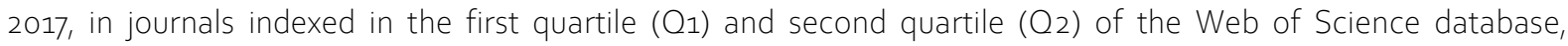
belonging to authors with affiliation in Brazilian institutions. Methodology: Communication journals in InCities (JCR) belonging to the first two quartiles were identified, then a search by journal title in WoSff was performed and subsequently the treatment and normalization of the data obtained. Results: A growth in scientific production in Communication is observed, in international projection, feeding a tradition in coauthorship with international networks. In the period from 2014 to 2017, they tended to publish mainly in English, against the usual
\end{abstract}




\section{$\mathrm{RDBCI}$}

collaborations that they had been maintaining with Spanish-speaking countries until 2014. Conclusion: We conclude, among other things, that the Capes evaluation system, quadrennial, may have influenced the scientific production in Communication in the studied interval.

\section{KEYWORDS}

Communication. Scientific production. Communication journals. Web of Science. Brazil

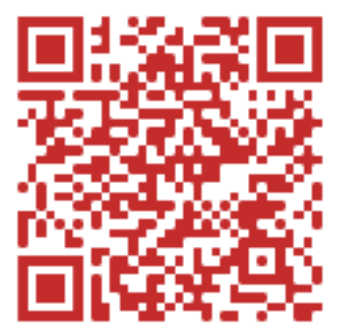

JITA: BJ. Communication 


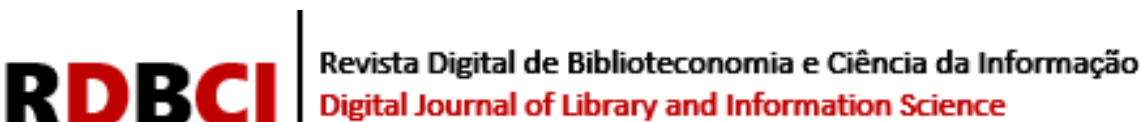

\section{INTRODUÇÃO}

De acordo com dados da Fundación Española para la Ciencia y la Tecnología (Fecyt) (2019), o Brasil está entre os 30 principais países na produção científica. A sua taxa de excelência, que indica que a percentagem das publicações de um país está incluída no conjunto dos $10 \%$ mais citados na sua área, é de $8,7 \%$. Por outro lado, a produção científica do país na base de dados Web of Science (WoS) tem crescido nos últimos anos.

Em 2018, a produção científica brasileira contabilizou 53.837 artigos, enquanto a percentagem de publicações em revistas de alto impacto (Q1) foi de 34,2\% (FECYT, 2019). Tendo em consideração que a atividade de investigação em Comunicação cresceu muito nos últimos anos, como indicado por estudos anteriores (PARK; LEYDESDORFF, 2009; KOIVISTO; THOMAS, 2011; MONTERO-DÍAZ et al., 2018), o presente trabalho visa estabelecer uma pesquisa em termos de produção científica nessa área, com base na identificação das revistas do primeiro e segundo quartis presentes na WoS. O objetivo é apreciar a evolução temporal dessa produção, conhecer a filiação institucional dos coautores, ver os temas abordados, a linguagem de publicação e as redes internacionais de países de coautoria.

A hipótese é que os sistemas de avaliação - por mérito docente - têm sido decisivos na produção científica em Comunicação durante o período estudado (2007-2017), com a produção crescendo à medida que esses requisitos aumentam em termos de bases de dados de referência, rankings e quartis de posicionamento. $\mathrm{O}$ aumento da produção que os estudos centrados no campo da Comunicação dentro da WoS apresentaram, levou à criação da categoria específica de Comunicação, o que faz da WoS uma ferramenta bibliométrica de referência na divulgação científica internacional para analisar o estado atual da produção científica em Comunicação no Brasil. Isso é reforçado pelo fato de ser uma plataforma que recolhe referências das principais publicações científicas em qualquer disciplina do conhecimento, tanto científico como tecnológico, humanista e sociológico, desde 1900 até o presente (FECYT, 2019).

\subsection{Impacto do sistema de avaliação na produção científica}

O aparecimento dos periódicos científicos na área da Comunicação começou nos anos 90, e foi a partir do ano 2000 que eles aumentaram em número, embora "a sua consolidação tenha sido difícil devido ao amadorismo dos seus criadores, à escassez de recursos econômicos e aos limitados processos bibliométricos, os investigadores que publicaram os seus artigos fizeram-no sem conhecer o valor de publicar num ou noutro" (CASTILLO et al., 2014, p.628).

No caso do Brasil, a avaliação da pós-graduação é de responsabilidade da Coordenação de Aperfeiçoamento do Pessoal de Educação Superior (Capes), instituição encarregada de avaliar os programas de pós-graduação no país. Entre as variáveis avaliadas encontra-se a produção científica dos professores e docentes, pesquisadores.

É um sistema que está em vigor há 40 anos, embora somente depois de 1988 tenham dado maior ênfase à produção científica de professores. A Capes presta atenção aos critérios de produtividade científica que podem servir para a seleção de professores por instituições de ensino superior, e sobretudo no impacto das avaliações institucionais por meio dos programas de pós-graduação, "de acordo com um perfil de produtividade, definição de áreas de concentração, linhas de investigação, e produzem efeitos de prestígio profissional e social que têm impacto no mapa do poder acadêmico nas instituições e na comunidade científica"(DIAS SOBRINHO, 2007, p.31). 


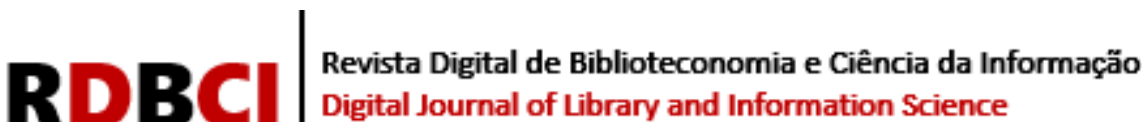

Setenta por cento da nota final que um programa de doutoramento pode obter depende da sua produção científica. Alguns autores propõem mudanças num sistema que deixa de lado aspectos mais qualitativos (CHEAP, 2019) e se concentra apenas em métodos quantitativos para avaliar as carreiras de doutoramento.

\subsection{Importância das bases de dados nos sistemas de avaliação docente}

No caso do Brasil, as bases de dados que estruturam e distribuem os resultados da investigação adquiriram grande importância nos sistemas de avaliação do pessoal docente, o que se refletiu na produção científica presente na base de dados da própria Web of Science (HOPPEN; VANZ, 2019). Essas bases possuem estudos centrados, durante anos, em repositórios (SANTOS, 2003), em indicadores bibliométricos da produção científica (MUGNAINI et al., 2004), na análise de citações da comunicação científica (VANZ; CAREGNATO, 2003) e na evolução das tendências da investigação científica e produção em nível mundial, tomando como referência as principais bases de dados multidisciplinares: WoS e a Scopus (DUDZIAK, 2010; DE FILIPPO; GARCÍA-ZORITA, 2020). Além da importância da ciência orientada para dados com estudos centrados na Scopus e na Web of Science (BUFREM et al., 2016), há que se destacar também a relevância dos estudos sobre indicadores de impacto científico das publicações, tais como o índice Hisrch $h$ (SILVA; GRÁCIO, 2017).

Os estudos com foco no campo da Comunicação têm vindo gradualmente a incidir em aspectos como a literatura científica nas Ciências da Informação (MUELLER, 2007), as redes de colaboração científica (BUFREM, 2010) e a relevância do cenário institucional (ARAÚJO; VALENTIM, 2019) e de redes sociais na divulgação científica (ALVES, 2011; PRÍNCIPE, 2013), assim como a produção científica sobre estudos de gênero em Ciências da Informação (SILVA, ZAPATA; OLIVERA, 2019; NEVES, 2018).

\subsection{Objetivos da pesquisa}

O objetivo geral é analisar a dinâmica da pesquisa na área da Comunicação, de autores com afiliação com o Brasil, por meio da produção científica indexada na Web of Science (WoS), durante o período de 2007 a 2017.

Os objetivos específicos são:

a) ver a evolução da produção científica nas principais revistas na WoS (Q1 e Q2);

b) analisar a filiação institucional das autorias;

c) identificar redes internacionais por meio da coautoria;

d) descrever os principais temas abordados na área de Comunicação; e

e) conhecer as revistas e o idioma em que mais se publica no âmbito da produção científica estudada.

\section{MÉTODO}

Primeiro, identificou-se quais as revistas da área de Comunicação presentes no InCites Journal Citation Reports (JCR), pertencentes aos dois primeiros quartis (Q1 e Q2), e que foram classificadas pelo Fator de Impacto (FI) em 2018. No total, foram recuperados 44 títulos de periódicos, 22 dos quais pertencentes ao Q1 e outros 22 ao Q2.

Subsequentemente, foi feita uma pesquisa avançada na Web of Science, para a qual 


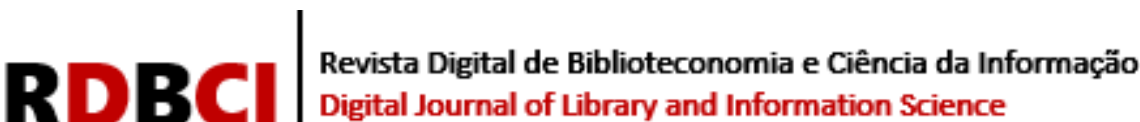

foram criados dois protocolos de pesquisa: o primeiro com o título das 22 revistas Q1 e o segundo com o título das 22 revistas Q2. Em ambos os casos, os títulos dos periódicos foram ligados por meio do operador booleano OR, o que permitiu a recuperação de artigos presentes em todos os periódicos listados. As fórmulas utilizadas na WoS para a criação do corpus da pesquisa em março de 2020 são detalhadas a seguir.

A primeira equação de busca foi usada para recuperar as publicações feitas nos 22 periódicos de Comunicação do primeiro quartil (Q1).

\section{Equação 1. Equação de busca WoS nas revistas de Comunicação (O1)}

$S O=$ (Communication Monographs) $O R S O=($ Communication Research) $O R$ SO $=$ (Communication Theory) $O R$ $S O=$ (Communication Methods and Measures) $O R$ SO=(Comunicar) OR $S O=$ (Digital Journalism) $O R$ $S O=($ Environmental Communication - A Journal of Nature and Culture) $O R$ SO $=$ (Human Communication Research) OR SO=(Information Communication \& Society) OR SO=(International Journal of Advertising) OR $S O=($ International Journal of Press-Politics) OR SO=(Journal of Advertising) OR SO=(Journal of Communication) $O R$ SO=(Journal of Computer-Mediated Communication) $O R$ SO=(Journalism) $O R$ $S O=($ Media Psychology) OR SO=(Mobile Media \& Communication) OR SO=(New Media \& Society) OR $S O=($ Political Communication) OR SO=(Public Opinion Quarterly) OR SO=(Public Understanding of Science) OR SO=(Science Communication)

A segunda equação de busca foi usada para recuperar as publicações feitas nos 22 periódicos de Comunicação do segundo quartil (Q2).

Equação 2. Equação de busca WoS nas revistas de Comunicação (O2)

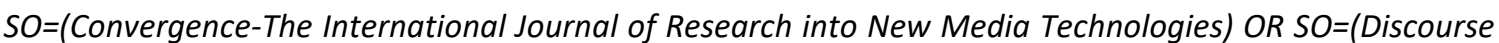
Studies) $O R$ SO=(European Journal Of Communication) $O R$ SO $=$ (Feminist Media Studies) OR SO $=($ Games And Culture) OR SO=(Health Communication) OR SO=(Journal Of Advertising Research) OR SO=(Journal Of Broadcasting \& Electronic Media) OR SO=(Journal Of Health Communication) OR SO=(Journal Of Social And Personal Relationships) OR SO=(Journalism \& Mass Communication Quarterly) OR SO=(Journal Of Media Psychology-Theories Methods And Applications) OR SO=(Journal Of Public Relations Research) OR $S O=(J o u r n a l i s m$ Practice) $O R$ SO=(Journalism Studies) OR SO=(Media Culture \& Society) OR SO=(MasS Communication And Society) $O R$ SO=(Public Relations Review) OR SO=(Policy And Internet) OR $S O=($ Profesional De La Informacion) $O R$ SO=(Research On Language And Social Interaction) OR $\mathrm{SO}=($ Telecommunications Policy)

A partir dessas equações de busca foram aplicados os filtros de temporalidade (2007-2017), tipo de documento (artigo) e país de origem (Brasil).

É importante esclarecer que o filtro oferecido pela WoS 'país de origem', é o país do vínculo institucional de pelo menos um dos autores da publicação. Deve-se ter em conta que cada artigo pode ter mais do que um autor e que cada um deles pode ter uma ou mais instituições de vínculo, assim como país de origem.

\subsection{Tratamento dos dados recuperados}

Os dados recuperados na WoS foram exportados para o editor de planilhas Excel, usado nos processos de limpeza, normalização e triagem. Os campos exportados e utilizados para esta análise foram: autores, afiliação dos autores, periódicos de publicação, ano de publicação, idioma do artigo e palavras-chave.

A partir disso, foram executados os seguintes passos: 


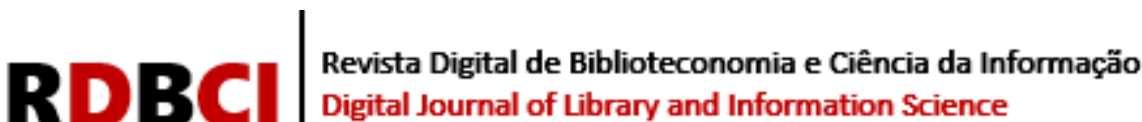

a) organização de colunas e células;

b) limpeza de dados não utilizados;

c) desagregação de autores e palavras-chave;

d) listagem e classificação dos dados; e

e) padronização dos termos.

Após a desagregação dos nomes dos autores, foram classificados um a um para definição de gênero (masculino e feminino) a partir do próprio nome. Embora subjetivo e com possibilidade de equívocos, acreditou-se ser importante verificar este dado, sobretudo para análises comparativas.

Depois procedeu-se ao desenvolvimento de representações visuais dos dados. Para tanto, foram escolhidas duas ferramentas diferentes: Excel e VOSviewer. As tabelas e gráficos foram desenvolvidos no Excel. Os grafos foram gerados a partir do software VOSviewer.

Contudo, no grafo que representa as palavras-chave, o thesaurus não foi utilizado devido ao grande número de termos. Nesse caso, a rede é representada pelas palavras-chave recuperadas diretamente dos artigos da $\mathrm{WoS}$, e não pelos termos padronizados.

\section{RESULTADOS}

Foram recuperados 68 artigos, publicados em 25 periódicos diferentes e com 177 autores envolvidos.

\subsection{Evolução da produção científica}

Figura 1. Quantidade de artigos recuperados por ano

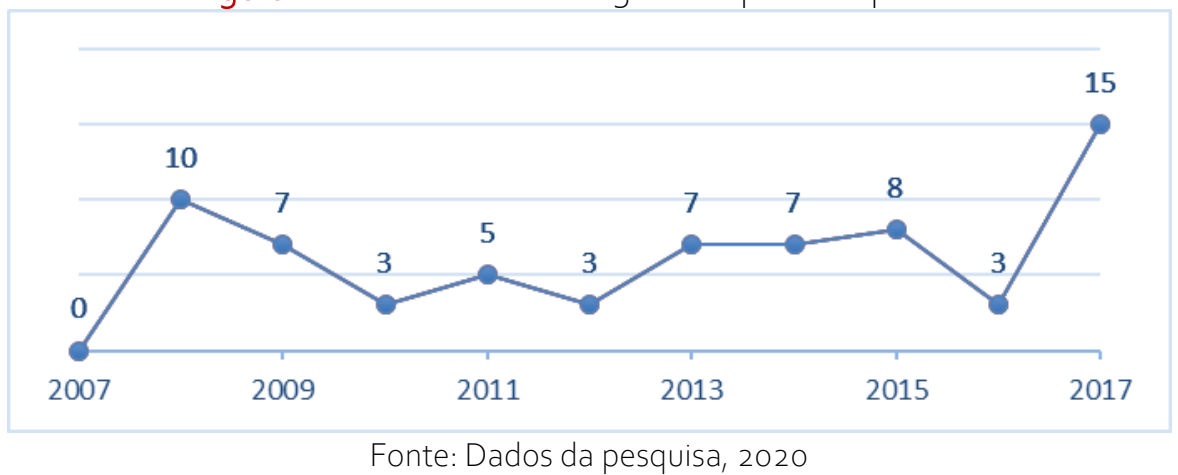

É possível considerar que os anos de 2010, 2012 e 2016 marcaram crises importantes no Brasil e/ou no mundo que podem justificar a diminuição da produção nesses anos.

Por outro lado, utilizando as avaliações da pós-graduação brasileira pela Capes, nos anos das avaliações trienais ou da quadrienal, podemos fazer algumas análises com o volume de publicações ao longo da década investigada. Houve 'publicação' das avaliações trienais nos seguintes anos: 2007, 2010 e 2013, passando à 'publicação' da primeira avaliação quadrienal ocorrida em 2017.

Nas duas últimas avaliações, houve aumento da produção no ano da publicação das avaliações de anos anteriores, logo o aumento da produção visível em 2013 e sobretudo em 2017 ocorrem nos primeiros anos de início de processos avaliativos. No entanto, a mesma lógica não ocorreu nos anos anteriores. Como já supramencionado, cenários distintos das 


\section{RDBCI|}

políticas da Capes e cenários político-econômicos também distintos podem ter influenciado fortemente o volume das publicações.

\subsection{Volume de difusão de artigos Q1 e Q2}

Como pode ser visto na Figura 2, o número de artigos no primeiro quartil (Q1) atingiu 59\%, com 40 artigos, em comparação com 41\% do segundo quartil (Q2), com 28 artigos.

Figura 2. Quantidade de artigos recuperados - Análise por quartil

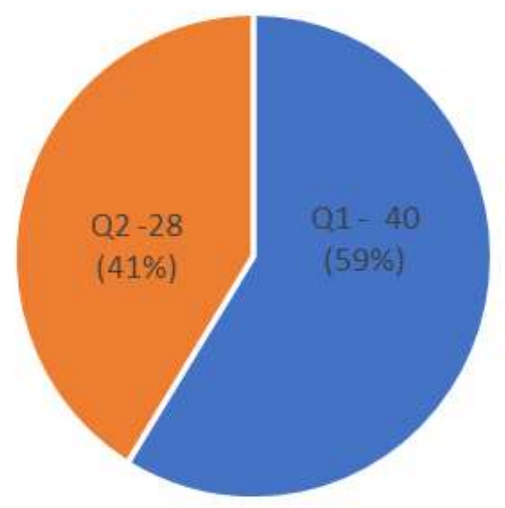

Fonte: Dados da pesquisa, 2020

Embora com número tímido de publicações, nota-se que a amostra para a década analisada apresenta que os autores obtiveram êxito em publicar sobretudo no primeiro quartil (Q1).

A Figura 3 mostra os dez periódicos pesquisados, nos quais houve maior presença de artigos assinados por autores de instituições brasileiras. Entre eles, dois periódicos espanhóis se destacam por concentrarem o maior número de artigos: Comunicar(Q1) e Profesional de la información (Q2). O primeiro publica em espanhol e o segundo em versão bilingue espanhol-inglês. As demais revistas apresentadas na Figura 3 são de origem inglesa ou estadunidense.

Figura 3. Revistas $\mathrm{Q}_{1}$ e Q2 em Comunicação com maior presença de autoria brasileira (2007-2017)

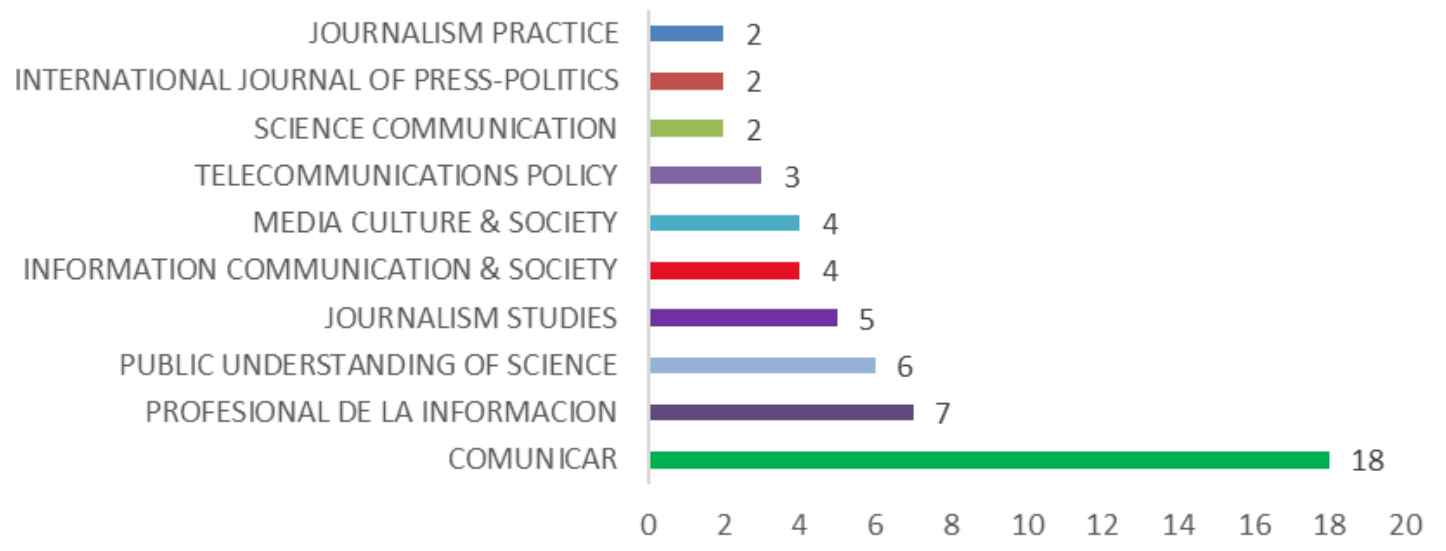

Fonte: Dados da pesquisa, 2020. 


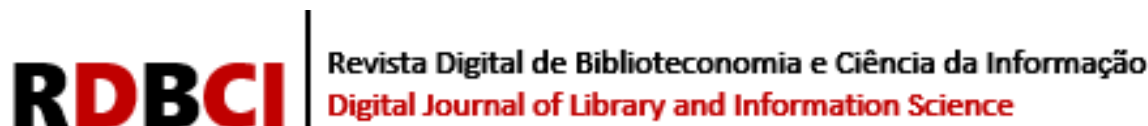

Com exceção da revista 'Telecommunications Policy', que disponibiliza, atualmente, o acesso fechado ao conteúdo, outros oito periódicos aparecem oferecendo a forma híbrida de acesso - artigos com acesso fechado e artigos com acesso aberto. São eles: 'Journalism Practice'; 'Information Communication \&Society'; 'Journalism Studies'; 'International Journal of Press-Politics'; 'Science Communication'; 'Media Culture \&Society'; 'Public Understanding of Science' e 'Profesional de la Información'. Além desses, a revista 'Comunicar' disponibiliza seus artigos de forma aberta.

\subsection{Afiliação institucional dos autores}

Dos 68 artigos analisados, observou-se que 54 (79\%) são em coautoria e 14 (21\%) foram publicados com autoria única, sendo o número médio de assinaturas por artigo de 3,1. Também foram identificadas as instituições de origem de todos os autores signatários dos artigos, totalizando 78 registros de diferentes instituições. Como é possível observar na Figura 4, as instituições que mais se destacam na afiliação institucional são brasileiras.

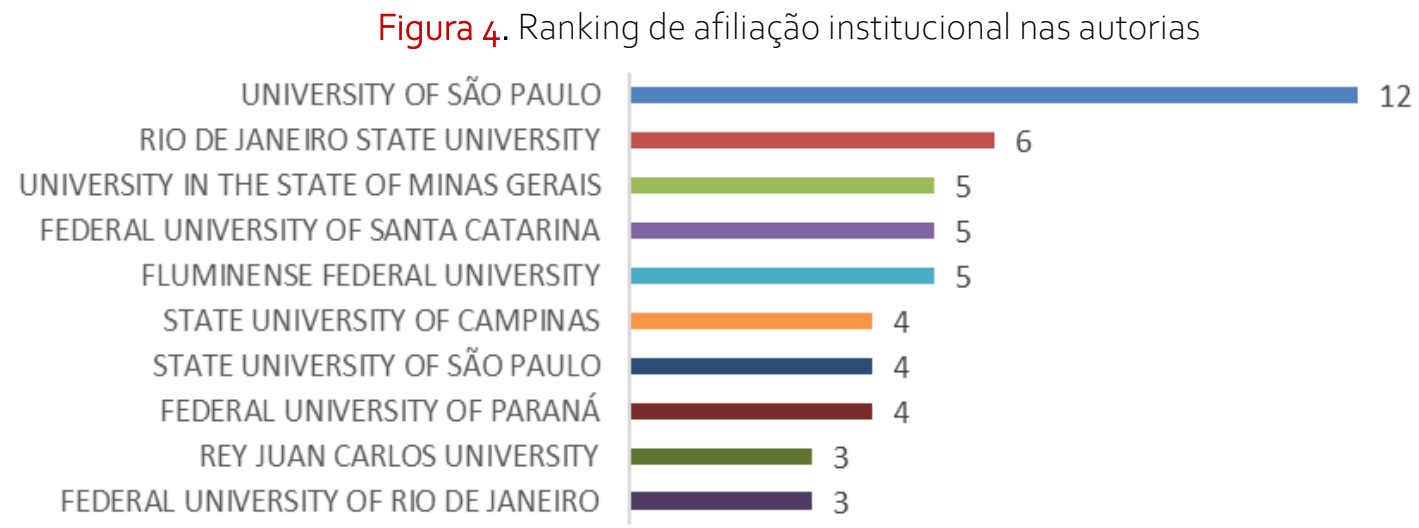

Identificadas as afiliações, procedeu-se à averiguação de quais são as instituições que mais aparecem nas assinaturas dos artigos. Entre as 78 instituições de afiliação dos autores, temos as dez com maior número de ocorrências, apresentadas na Figura 4. Aparecem na sequência, com um total de duas ocorrências cada, as seguintes instituições: African Centre for Media Excellence; Anadolu University; Austrian Academy of Sciences; Ben-Gurion University of the Negev; Cairo University; California State University; California State University System; Colorado State University; Lomonosov Moscow State University; University of Santiago; University of Brasília; University of Coimbra; Federal University of Bahia; Federal University of Rio Grande do Norte; Federal University of Rio Grande do Sul; University of Bucharest; University of Colorado Boulder; University of Colorado System; University of Munich; University of Texas at Austin; University of Texas System; e University of the Sunshine Coast. Em seguida, de forma pulverizada, temos 46 outras instituições que aparecem com apenas uma ocorrência.

Esses dados permitem-nos ver que, para além da Universidade de São Paulo, não existem centros que se destaquem dos outros de forma significativa em termos de filiação institucional das autorias. 


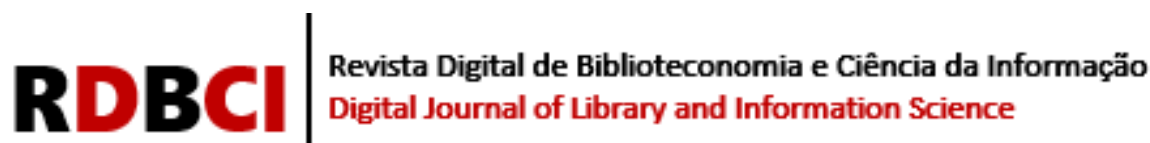

\subsection{Redes de coautoria por países}

Observa-se como a tendência vem mudando ao longo dos anos. Entre 2010 e 2014, predominou a colaboração com China, Rússia, Estados Unidos e países de língua espanhola, como Espanha, Chile e México. A situação muda a partir de 2014, dando origem a uma colaboração de coautores com autores da Inglaterra, Cuba, República Tcheca, França, Irlanda do Norte, África do Sul e Holanda.

Figura 5. Evolução da coautoria internacional (representada por países)

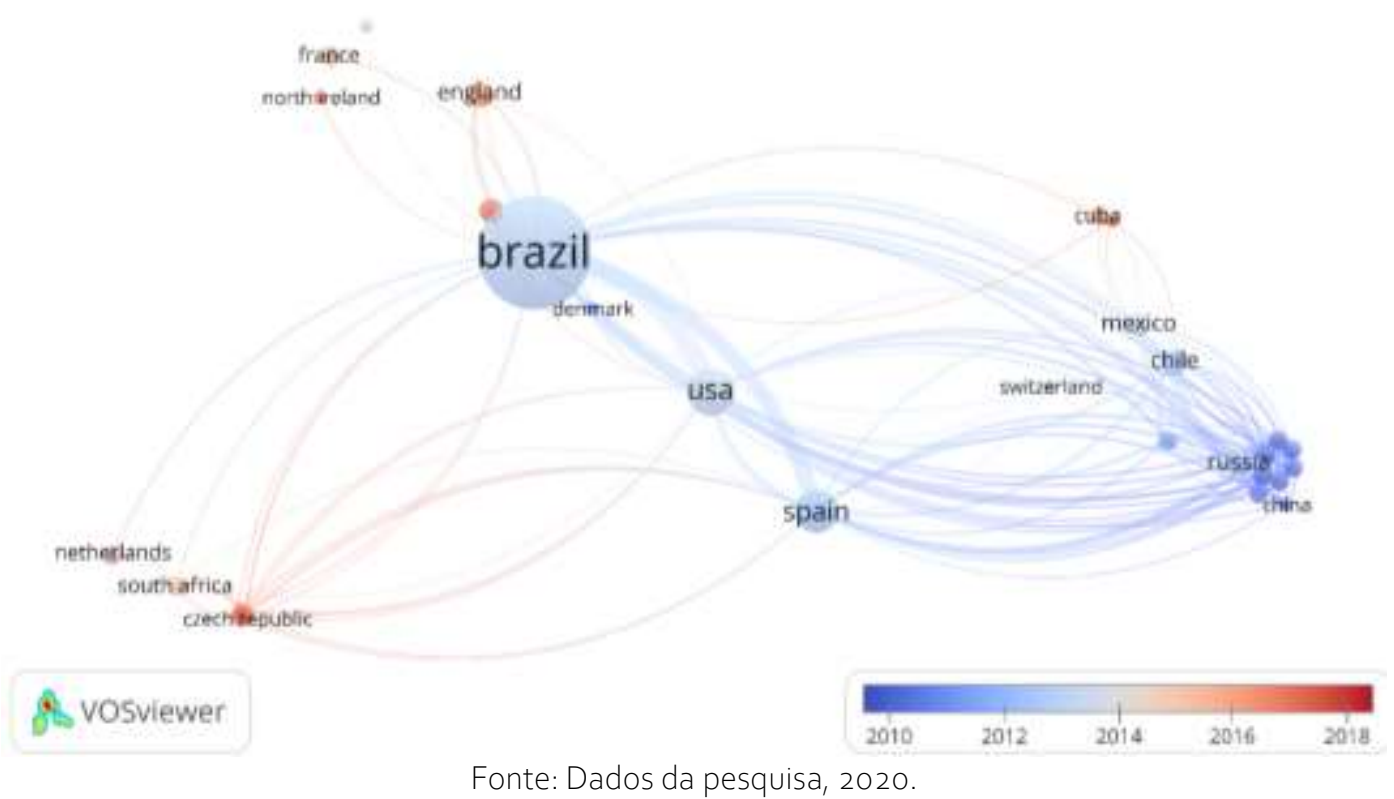

Ao observar o idioma de publicação dos artigos analisados, verifica-se que dos 68 artigos analisados, 50 são em inglês (74\%), 15 são em espanhol (22\%) e 3 em português (4\%).

\subsection{Principais autores, temáticas e palavras-chave}

As palavras-chave mais utilizadas podem ser vistas na Figura 6. Para tal, foi utilizada uma escala temporal, de modo que a escala anual, presente no canto inferior das figuras gráficas, representa o ano médio em que ambas as palavras-chave tiveram a maior frequência de utilização e os países tiveram os documentos mais publicados. Note-se que esse não é o primeiro ou o último ano, é o ano médio, ou seja, um cenário com todas as palavras nos anos onde suas ocorrências foram mais acentuadas. 


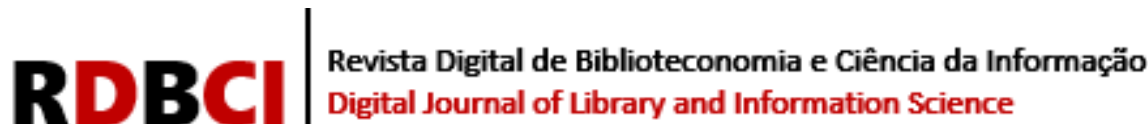

Figura 6. Grafo da maioria de palavras-chave recorrentes

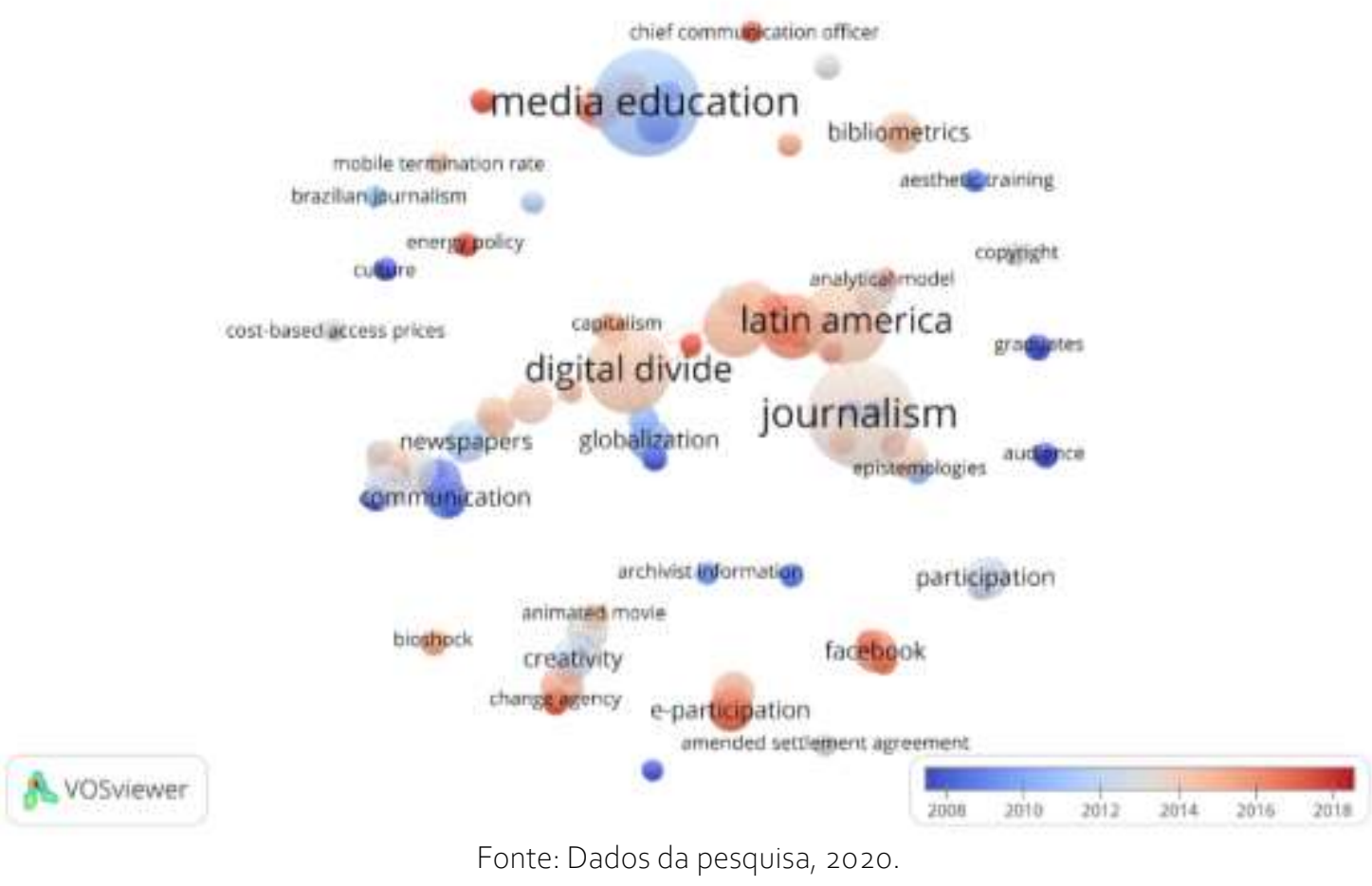

Nos artigos de autores brasileiros, verifica-se que os termos que mais têm predominado são: jornalismo, educação midiática, dividendo digital, América Latina e modelo analítico. Essas são palavras que têm sido mantidas ao longo dos anos, e às quais foram recentemente acrescentadas outras, tais como: Facebook, e-participação ou bibliometria. Ao mesmo tempo, há termos que eram amplamente utilizados e que vêm perdendo uso, tais como: comunicação, jornais, globalização e audiência.

A escala de ano presente no canto inferior das figuras dos grafos (VOSviewer) representa o ano médio em que tanto as palavras-chave tiveram maior frequência de uso como os países tiveram mais documentos publicados. Cabe lembrar que não é o primeiro ano ou o último ano, é o ano médio, ou seja, considerando uma média diante de todas as palavras e suas aparições e todos os países e suas aparições. Quanto mais vermelho, mais recente foi esse uso/envolvimento respectivamente.

Analisando os tópicos abordados nos artigos por palavras-chave, foram identificados 323 temas normalizados pelas autoras do estudo. Os temas mais importantes, apresentados na Figura 7, foram: jornalismo, educação midiática, dispositivos móveis, brecha digital, América Latina, análise de conteúdo e internet. A partir da sétima posição, têm-se 28 temas que apareceram somente duas vezes e os demais somente uma vez. 


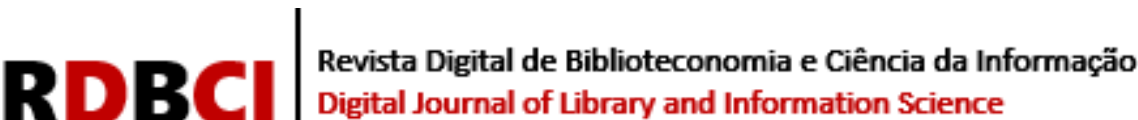

Figura 7. Temas (normalizados) mais frequentes dos artigos
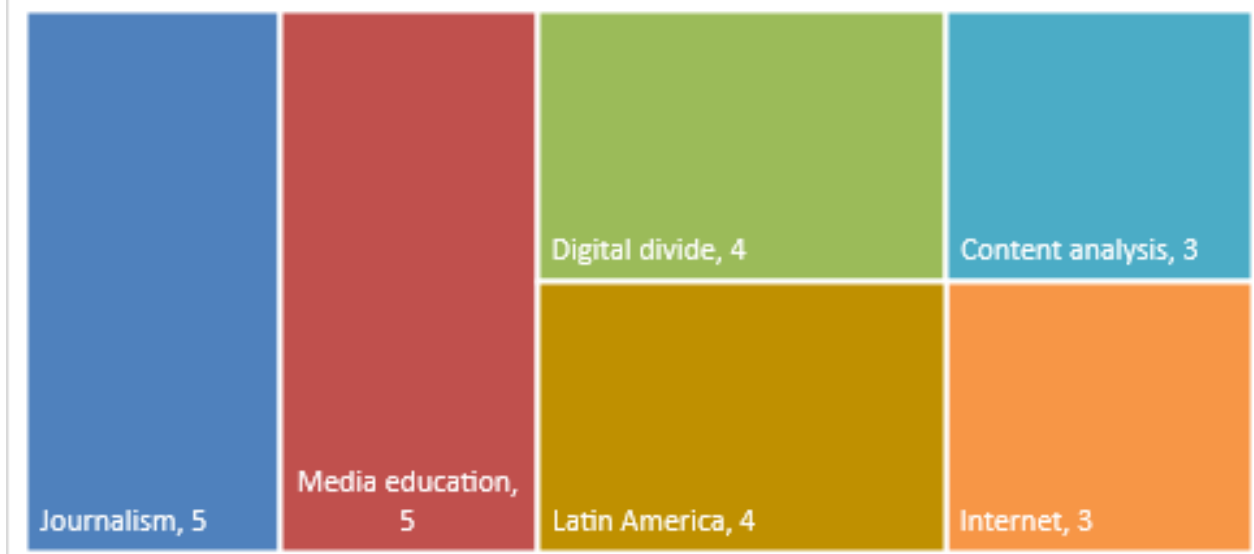

Fonte: Dados da pesquisa, 2020

Quanto às autorias, entre os 177 autores que assinam os artigos analisados, no que se refere ao gênero, há uma predominância de homens $(110-62 \%)$ em relação as mulheres $(67-38 \%)$. Com relação ao volume de produção desses mesmos autores, foram identificados 23 autores com dois ou mais artigos publicados entre os artigos analisados. Percebe-se que $87 \%$ dos autores assinaram apenas um artigo e a média de assinatura no caso dos outros $13 \%$ é de 2,3 artigos. A autora de maior destaque foi Claudia Mellado, com quatro artigos.

\section{DISCUSSÃO E CONCLUSÕES}

Os resultados desta investigação estão de acordo com estudos anteriores (PARK; LEYDESDORFF, 2009; KOIVISTO; THOMAS, 2011; MONTERO-DÍAZ et al., 2018), demonstrando que a atividade de investigação em Comunicação cresceu nos últimos anos e de maneira muito significativa em 2017.

Os resultados obtidos permitem validar a hipótese de que o sistema de avaliação da Capes pode ter influenciado na produção científica em Comunicação durante o período estudado, em linha com as observações de autores como Dias Sobrinho (2007). Foi observada uma predominância da produção científica no primeiro quartil (Q1), como recomendado por agências de fomento e instituições de avaliação como CNPq e Capes, respectivamente. Além do local de publicação da produção cientifica em Comunicação, percebe-se uma tradição em coautoria com redes internacionais, de acordo com os critérios valorados pela Capes. Observa-se como essas relações têm sido fluidas com países como Estados Unidos da América, Espanha, China, Rússia, Chile e México.

Acredita-se que um dos fatores que impulsionam essa dinâmica de colaboração internacional seja o sistema de avaliação da Capes aliada as políticas de incentivo, que havia para pesquisas, estágios e intercâmbios internacionais, até o ano de 2016.

Em termos de idioma, no intervalo de 2014 a 2017 tenderam a publicar em periódicos onde os resultados da pesquisa são divulgados sobretudo em inglês, como mostra o fato de $74 \%$ dos artigos publicados serem veiculados nesse idioma.

Tem havido um crescimento na divulgação científica em Comunicação por autores brasileiros durante o período estudado, o que corresponde ao aumento geral da produção científica nos últimos anos, como indicado pela Fecyt (2019). 


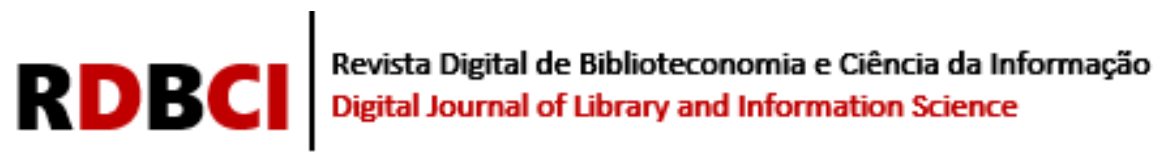

Na produção cientifica analisada há maior presença de autores (homens) do que de autoras (mulheres) e com predomínio das universidades brasileiras como filiação institucional de autoria.

Entre os artigos analisados, o interesse por certos temas foi concentrado. Os assuntos mais abordados nos artigos têm sido o jornalismo, a educação midiática e a divisão digital. Esses dados refletem uma realidade muito diferente entre a Europa e a América Latina, que, por outro lado, marcam a agenda de investigação em Comunicação de autores brasileiros. A América Latina vem trabalhando há anos na utilização de tecnologias e meios de comunicação em contextos educativos para reduzir uma brecha digital que exclui milhões de pessoas que não têm internet.

\section{CRediT}

RECONHECIMENTOS: Não é aplicável.

FINANCIAMENTO: Não é aplicável.

CONFLITOS DE INTERESSE: Os autores certificam que não têm interesse comercial ou associativo que represente um conflito de interesses em relação ao manuscrito.

APROVAÇÃO ÉTICA: Não é aplicável.

DISPONIBILIDADE DE DADOS E MATERIAL: Os dados não foram organizados de forma a serem disponibilizados. CONTRIBUIÇÕES DOS AUTORES: Conceituação, Curadoria de Dados, Análise Formal, Aquisição de Financiamento, Investigação, Metodologia, Administração de Projetos, Recursos, Software, Supervisão, Validação, Visualização, Escrita - rascunho original, Escrita - revisão \& edição: LUCAS, E. R. O; AGUADO-GUADALUPE, M. G. A.; HERRERO-CURIEL, E.

\section{REFERÊNCIAS}

ALVES, Leticia. Informação e os sistemas de comunicação científica na Ciência da Informação. DataGramaZero, v.12, n.3, 2011. Disponível em: http://hdl.handle.net/20.500.11959/brapci/7379. Acesso em: 10 ago. 2021.

ARAÚJO, Carlos Alberto Ávila; VALENTIM, Marta Ligia Pomim. A ciência da informação no Brasil: mapeamento da pesquisa e cenário institucional. Bibliotecas. Anales de Investigación, Cuba, v. 15, n.2, p. 232-259, 2019. Disponível em: http://hdl.handle.net/20.500.11959/brapci/112206. Acesso em: 10 ago. 2021.

BUFREM, Leilah. Revisando vertentes na literatura em Ciência da Informação no Brasil. Tendências da Pesquisa Brasileira em Ciência da Informação, João Pessoa, v.3, n.1, p.127-151, 2010. Disponível em: http://hdl.handle.net/20.500.11959/brapci/119348. Acesso em: 10 ago. 2021.

BUFREM, Leilah et al. Produção internacional sobre ciência orientada da dados: análise dos termos data science e e-science na Scopus e na Web of Science. Informação \& Informação, Londrina, v.21, n.2, p. 40-67, 2016. Disponível em:

https://doi.org/10.5433/1981-8920-2016v21n2p40. Acesso em: 25 set. 2020.

CASTILLO, Antonio et al. Revista de Comunicación en España. Una aproximación histórica. Historia y Comunicación Social, Madrid, v. 19, Número especial (febrero): La comunicación 


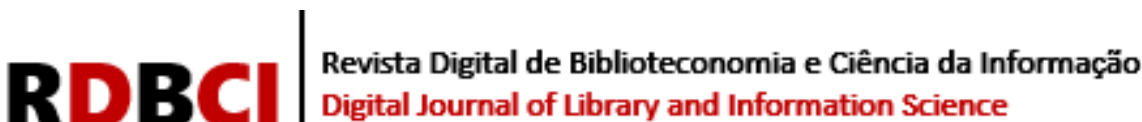

en la profesión en la universidad de hoMORENAy, p.621-630, 2014. Disponível em: https://doi.org/10.5209/rev hics.2014.v19.45053. Acesso em: 22 ago. 2020.

DE FILIPPO, Daniela; GARCÍA-ZORITA, Carlos (ed.). Actividad investigadora de las universidades españolas (VII). Espanha: Observatorio IUNE, 2020.

DIAS SOBRINHO, José. Evaluación de la Educación Superior en Brasil: políticas y prácticas. Revista Complutense de Educación, v. 18, n. 2, p. 29 - 44, ago. 2007. Disponível $\mathrm{em}$ : https://revistas.ucm.es/index.php/RCED/article/view/RCED0707220029A. Acesso em: 10 ago.2021.

DUDZIAK, Elisabeth. Competência informacional: análise evolucionária das tendencias da pesquisa e produtividade científica em âmbito mundial. Informação \& Informação, Londrina v.15, n.2, p.1-22, 2010. Disponível em: http://dx.doi.org/10.5433/1981-8920.2010v15n2p1. Acesso em: 10 ago.2021.

FECYT. Producción científica en WOS. Espanha: Observatório Español de I+D+I, 2019. Disponível em: https://bit.ly/3y9Y4sW. Acesso em: 10 ago. 2020.

HOPPEN, Natascha; VANZ, Samile. Primeiras impressões da pesquisa autodenominada Estudos de Gênero em uma base de dados internacional e multidisciplinar. In: ENCONTRO NACIONAL DE PESQUISA EM CIÊNCIA DA INFORMAÇÃO, 20., 2019, Florianópolis. Anais [...]. Florianópolis, 2019. p. 1-9. Disponível em: https://conferencias.ufsc.br/index.php/enancib/2019/paper/view/592. Acesso em: 22 ago. 2020.

KOIVISTO, Juha; THOMAS, Peter. Mapping communication and media research: Conjunctures, institutions, challenges. Finlândia: Tampere University Press, 2011. Disponível em: http://urn.fi/URN:ISBN:978-951-44-8144-4. Acesso em: 10 ago. 2021

MONTERO-DÍAZ, Julio et al. A science mapping analysis of 'Communication' WoS subject category (1980-2013). Comunicar, Huelva, v.26, n.55, p. 81-91, 2018. Disponível em: https://doi.org/10.3916/c55-2018-08. Acesso em: 10 ago. 2020.

MUELLER, Susana. Literatura científica comunicação científica e ciência da informação. In: TOUTAIN, Lídia María. Para entender a Ciência da Informação. Salvador: Editora da Universidade Federal Da Bahia, 2007. p.125-144. Disponível em:

https://repositorio.ufba.br/ri/bitstream/ufba/145/1/Para $\% 20$ entender $\% 20 \mathrm{a} \% 20$ ciencia $\% 20 \mathrm{da} \%$ 20informacao.pdf. Acesso em: 10 ago.2021.

MUGNAINI, Rogério; JANNUZZI, Paulo; QUONIAM, Luc. Indicadores bibliométricos da produção científica brasileira: uma análise a partir da base Pascal. Ciência da Informação, Brasília, v. 33, n. 2, p. 123-131, 2004. Disponível em: https://doi.org/10.1590/S0100-19652004000200013. Acesso em: 08 ago. 2020.

NEVES, Tatiely Mayara de Oliveira. A mulher e a comunicação científica: uma questão muito além do gênero. Múltiplos Olhares em Ciência da Informação, Minas Gerais v. 8, n. 2, p. 1-8, 2018. Disponível em: https://periodicos.ufmg.br/index.php/moci/article/view/16889. Acesso em: 10 ago. 2021. 


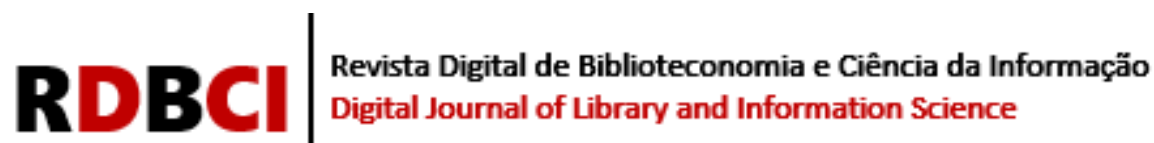

PARK, Han Woo; LEYDESDORFF, Loet. Knowledge linkage structures in communication studies using citation analysis among communication journals. Scientometrics, Holanda, v.81, n.1, p.157-175, 2009. Disponível em: https://doi.org/10.1007/s11192-009-2119-y. Acesso em: 10 ago. 2020.

PRÍNCIPE, Eloisa. Fronteiras da Ciência da Informação. In: ALBAGLI, Sarita. Fronteiras da Ciência da Informação. Brasília: IBICT, p. 196-216, 2013. Disponível em: http://livroaberto.ibict.br/handle/1/1020. Acesso em: 10 ago.2021.

SANTOS, Raimundo Nonato. Produção científica: por que medir? O que medir? Revista Digital de Biblioteconomia e Ciência da Informação, Campinas, v.1, n.1, p.22-38, 2003. Disponível em: https://doi.org/10.20396/rdbci.v1i1.2087. Acesso em: 10 ago. 2021

SILVA, Deise; GRÁCIO, María Cláudia. Indice h de Hirsch: análise comparativa entre as bases de dados Scopus, Web of Science e Google Acadêmico. Em Questão, Porto Alegre, v.23, p. 196-212, 2017. Disponível em: http://dx.do.org/10.19132/1808-5245230.196-212. Acesso em: 10 ago. 2020.

SILVA, Ester; ZAPATA, Cristian; OLIVEIRA, Hamilton. Análise da produção científica sobre gênero na Ciência da Informação. In: ENCONTRO NACIONAL DE PESQUISA EM CIÊNCIA DA INFORMAÇÃO, 20., 2019, Florianópolis. Anais [...]. Florianópolis, 2019. Disponível em: https://conferencias.ufsc.br/index.php/enancib/2019/paper/view/866. Acesso em: 22 ago. 2020.

VANZ, Samile; CAREGNATO, Sônia. Estudos de Citação: uma ferramenta para entender a comunicação científica. Em Questão, Porto Alegre, v.9, n.2, p. 295-307, 2003. Disponível em: https://seer.ufrgs.br/EmQuestao/article/view/75/35. Acesso em: 10 ago. 2021 


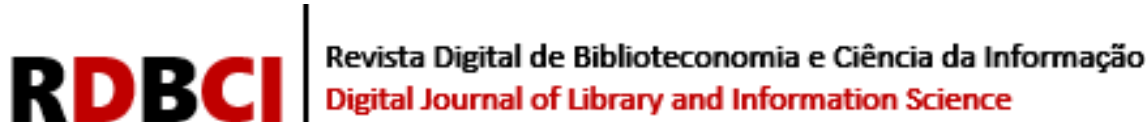

